\title{
On Common Fixed Point Theorem of Four Self Maps in a Fuzzy Metric Space
}

\author{
Manthena Prapoorna, Manchala Rangamma \\ Department of Mathematics, Osmania University, Hyderabad, India \\ Email:m_prapoorna@yahoo.com
}

Received 27 January 2016; accepted 28 March 2016; published 31 March 2016

Copyright $@ 2016$ by authors and Scientific Research Publishing Inc.

This work is licensed under the Creative Commons Attribution International License (CC BY). http://creativecommons.org/licenses/by/4.0/

c) (i) Open Access

\begin{abstract}
In the present paper, we show that there exists a unique common fixed point for four self maps in a fuzzy metric space where two of the maps are reciprocally continuous and the other two maps are $z$-asymptotically commuting.
\end{abstract}

\section{Keywords}

T-Norm, Fuzzy Metric Space, Reciprocally Continuous, Z - Asymptotically Commuting Maps

\section{Introduction}

L. Zadeh's [1] investigation of the concept of fuzzy set in the year 1965, has led to a rich growth of fuzzy mathematics. Today, it has become a well-accepted system to embrace upon uncertainties springing in numerous physical situations. The theory of fixed point equations is one of the extrusive basic tools to exploit various physical formulations. Theorems on fixed points in fuzzy mathematics are emerging with flourishing hope and vital certainty.

Many authors have introduced the concept of fuzzy metric space in various ways and have shown that every metric induces a fuzzy metric. There have been several endeavors to formulate fixed point theorems in fuzzy mathematics. In 1975, Kramosil and Michalek [2] generalized the statistical metric space and defined the fuzzy metric space which was later modified by George and Veeramani [3] [4] by introducing the concept of continuous t-norms. Recently, many researchers [5]-[9] have enormously developed the theory by studying various aspects of the theory and extending the concept of fuzzy metric through applying several contractive, expansive, continuity and compatibility conditions on the fuzzy metric and producing different results.

Pant [10] introduced the notion of reciprocally continuous mappings and established a fixed point theorem. S. N. Mishra, Nilima Sharma, S. L. Singh [11] defined z-asymptotically commuting maps in fuzzy metric spaces which may be seen as a comparable formulation given by Trivari-Singh [12] in metric spaces. These mappings 
are more general than commuting and weakly commuting maps.

The aim of this paper is to show that the self maps in a fuzzy metric space satisfying certain properties and inequalities possess a common fixed point which is unique.

\section{Preliminaries}

Here, we shall recall some prefaces:

Definition 2.1 ([13]): A binary operation $*:[0,1] \times[0,1] \rightarrow[0,1]$ is said to a continuous t-norm if $([0,1], *)$ is an abelian topological monoid with unit $1 \ni x * y \leq z * w$ whenever $x \leq z \quad \& \quad y \leq w \quad(x, y, z, w \in[0,1])$.

2.1 $(\alpha)$ Basic continuous t-norms are:

- $a * b=\min \{a, b\} \quad$ (minimum t-norm)

- $a * b=a b$ (product t-norm)

- $a * b=\max \{a+b-1,0\} \quad$ (Lukasiewicz t-norm)

- $a * b=\left\{\begin{array}{ll}\min \{a, b\} & \text { if } \max (a, b)=1 \\ 0 & \text { otherwise }\end{array}\right.$ (weakest t-norm, the drastic product)

Definition 2.2 ([3]): Let $\mathrm{X}$ be any non-empty set, * is a continuous t-norm and $\mathrm{M}$ is a fuzzy set on $\mathrm{X} \times \mathrm{X} \times$ $(0, \infty)$ satisfying

a) $\mathrm{M}(x, y, t)>0$

b) $\mathrm{M}(x, y, t)=1 \Leftrightarrow x=y$

c) $\mathrm{M}(x, y, t)=\mathrm{M}(y, x, t)$

d) $\mathrm{M}(x, y, t) * \mathrm{M}(y, z, s) \leq \mathrm{M}(x, z, t+s)$

e) $\mathrm{M}(x, y,):.(0, \infty) \rightarrow(0,1]$ is continuous where $x, y, z \in \mathrm{X}, \quad s, t>0$

Here, $\mathrm{M}(x, y, t)$ denotes the degree of nearness between $x, y$ with respect to " $t$ ".

- Grabiec ([14]) had shown that $\mathrm{M}(x, y,$.$) is non-decreasing \forall x, y \in \mathrm{X}$.

Definition 2.3 ([3]): A sequence $\left\{x_{n}\right\}$ in a fuzzy metric space $(\mathrm{X}, \mathrm{M}, *)$ said to converge to $x \in \mathrm{X} \Leftrightarrow$ $\mathrm{M}\left(x_{n}, x, t\right) \rightarrow 1$ as $n \rightarrow \infty$.

Definition 2.4 ([3]): A sequence $\left\{x_{n}\right\}$ in a fuzzy metric space $(\mathrm{X}, \mathrm{M}, *)$ is said to be a Cauchy sequence if for each $\varepsilon>0, t>0$, there exists $n_{0} \in \mathrm{N}$ such that $\mathrm{M}\left(x_{n}, x_{m}, t\right)>1-\varepsilon$ for all $n, m \geq n_{0}$.

Definition 2.5 ([3]): If every Cauchy sequence in a fuzzy metric space $\mathrm{X}$ is convergent, then $\mathrm{X}$ is said to be complete.

Definition 2.6 ([10]): Two self maps A and B of a fuzzy metric space (X, M,*) are said to be reciprocally continuous on $\mathrm{X}$ if

$$
\lim _{n \rightarrow \infty} \mathrm{AB} x_{n}=\mathrm{A} x \text { and } \lim _{n \rightarrow \infty} \mathrm{BA} x_{n}=\mathrm{B} x
$$

whenever $\left\{x_{n}\right\}$ is a sequence in $\mathrm{X}$ such that

$$
\lim _{n \rightarrow \infty} \mathrm{A} x_{n}=x \text { and } \lim _{n \rightarrow \infty} \mathrm{B} x_{n}=x
$$

for some $x \in \mathrm{X}$.

Definition 2.7 ([11]): Two self maps A and B of a fuzzy metric space $X$ are said to be z-asymptotically commuting if and only if

$$
\lim _{n \rightarrow \infty}\left(\mathrm{AB} x_{n}, \mathrm{BA} x_{n}, t\right)=1
$$

whenever $\left\{x_{n}\right\}$ is a sequence in $\mathrm{X}$ such that

$$
\lim _{n \rightarrow \infty} \mathrm{A} x_{n}=\lim _{n \rightarrow \infty} \mathrm{B} x_{n}=\mathrm{z}
$$

for some $z \in X$ and $\forall t>0$.

Lemma 2.8 ([14]): Let $(X, M, *)$ be a fuzzy metric space. If there exists $q \in(0,1)$ such that $\mathrm{M}(x, y, q t) \geq \mathrm{M}(x, y, t)$ for all $x, y \in \mathrm{X}$ and $t>0$, then $x=y$.

Succeeding the Grabiec's approach to fuzzy contraction principle, Mishra. S. N., Nilima Sharma, Singh. S. L. [11] had obtained common fixed point theorem for asymptotically commuting maps in fuzzy metric spaces. 
Theorem 2.9 ([11]): Let $(\mathrm{X}, \mathrm{M}, *)$ be a complete fuzzy metric space with $t * t \geq t, t \in[0,1]$ and $\mathrm{P}, \mathrm{Q}: \mathrm{X} \rightarrow \mathrm{X}$. If there exist continuous maps $\mathrm{S}, \mathrm{T}: \mathrm{X} \rightarrow \mathrm{X}$ and a constant $k \in(0,1)$ such that

1) $\mathrm{ST}=\mathrm{TS}$

2) $\{\mathrm{P}, \mathrm{S}\}$ and $\{\mathrm{Q}, \mathrm{T}\}$ are asymptotically commuting pairs

3) $\mathrm{PT}(\mathrm{X}) \cup \mathrm{QS}(\mathrm{X}) \subset \mathrm{ST}(\mathrm{X})$

4) $\mathrm{M}(\mathrm{P} x, \mathrm{Q} y, k t) \geq \mathrm{M}(\mathrm{S} x, \mathrm{~T} y, t) * \mathrm{M}(\mathrm{P} x, \mathrm{~S} x, t) * \mathrm{M}(\mathrm{Q} y, \mathrm{~T} y, t) * \mathrm{M}(\mathrm{P} x, \mathrm{~T} y, \alpha t) * \mathrm{M}(\mathrm{Q} y, \mathrm{~S} x,(2-\alpha) t)$

for all $x, y \in \mathrm{X}, t>0$ and $\alpha \in(0,2)$ then $\mathrm{P}, \mathrm{Q}, \mathrm{S}, \mathrm{T}$ have a unique common fixed point.

\section{Main Results}

Theorem 3.1: Let $(\mathrm{X}, \mathrm{M}, *)$ be a complete fuzzy metric space $\& *$ be any of the continuous t-norms given in $2.1(\alpha)$ and let $\mathrm{A}, \mathrm{B}, \mathrm{S}, \mathrm{T}$ be self maps of X satisfying

- The pair $\{\mathrm{A}, \mathrm{S}\}$ is reciprocally continuous

- The pair $\{\mathrm{B}, \mathrm{T}\}$ is $z$-asymptotically commuting

- The pairs $\{\mathrm{B}, \mathrm{S}\}$ and $\{\mathrm{T}, \mathrm{S}\}$ commute with each other

- $\mathrm{M}(\mathrm{A} x, \mathrm{~B} y, k t) \geq \frac{1}{2}\{\mathrm{M}(\mathrm{A} x, \mathrm{~T} y, t)+\mathrm{M}(\mathrm{S} x, \mathrm{~B} y, t)\} * \max \{\mathrm{M}(\mathrm{A} x, \mathrm{~S} x, t), \mathrm{M}(\mathrm{B} y, \mathrm{~T} y, t), \mathrm{M}(\mathrm{S} x, \mathrm{~T} y, t)\}$

where $x, y \in \mathrm{X}, t, k \in(0,1)$, then $\mathrm{A}, \mathrm{B}, \mathrm{S}$, T have a unique common fixed point in $\mathrm{X}$.

Proof: $\{\mathrm{A}, \mathrm{S}\}$ is reciprocally continuous:

$\Rightarrow \lim _{n \rightarrow \infty} \mathrm{AS} x_{n}=\mathrm{A} u$ and $\lim _{n \rightarrow \infty} \mathrm{SA} x_{n}=\mathrm{S} u$

whenever $\left\{x_{n}\right\}$ is a sequence in $\mathrm{X}$ such that

$$
\lim _{n \rightarrow \infty} \mathrm{A} x_{n}=u \text { and } \lim _{n \rightarrow \infty} \mathrm{S} x_{n}=u \text { for some } u \in \mathrm{X}
$$

$\{\mathrm{B}, \mathrm{T}\}$ is z-asymptotically commuting:

$\Rightarrow \lim _{n \rightarrow \infty}\left(\mathrm{TB} y_{n}, \mathrm{BT} y_{n}, t\right)=1$

whenever $\left\{y_{n}\right\}$ is a sequence in $\mathrm{X}$ such that

$$
\lim _{n \rightarrow \infty} \mathrm{B} y_{n}=\lim _{n \rightarrow \infty} \mathrm{T} y_{n}=z \text { for some } z \in \mathrm{X}
$$

- To prove that $u=z$ :

Put $x=x_{n}$ and $y=y_{n}$ in (1), we get

$\mathrm{M}\left(\mathrm{A} x_{n}, \mathrm{~B} y_{n}, k t\right) \geq \frac{1}{2}\left\{\mathrm{M}\left(\mathrm{A} x_{n}, \mathrm{~T} y_{n}, t\right)+\mathrm{M}\left(\mathrm{S} x_{n}, \mathrm{~B} y_{n}, t\right)\right\} * \max \left\{\mathrm{M}\left(\mathrm{A} x_{n}, \mathrm{~S} x_{n}, t\right), \mathrm{M}\left(\mathrm{B} y_{n}, \mathrm{~T} y_{n}, t\right), \mathrm{M}\left(\mathrm{S} x_{n}, \mathrm{~T} y_{n}, t\right)\right\}$

Letting $n \rightarrow \infty$, we have

$\mathrm{M}(u, z, k t) \geq \frac{1}{2}\{\mathrm{M}(u, z, t)+\mathrm{M}(u, z, t)\} * \max \{\mathrm{M}(u, u, t), \mathrm{M}(z, z, t), \mathrm{M}(u, z, t)\} \quad$ (Since from (2) and (3))

$\mathrm{M}(u, z, k t) \geq \frac{1}{2}\{\mathrm{M}(u, z, t)+\mathrm{M}(u, z, t)\} * \max \{1,1, \mathrm{M}(u, z, t)\}$ (Since $\left.\mathrm{M}(x, y, t)=1 \Leftrightarrow x=y\right)$

$\mathrm{M}(u, z, k t) \geq \mathrm{M}(u, z, t) * 1$

$\mathrm{M}(u, z, k t) \geq \mathrm{M}(u, z, t)$

$\Rightarrow u=z \quad$ (from lemma 2.8)

i.e., we can find a $z \in \mathrm{X}$ such that (2) and (3) holds simultaneously.

Consider (3)

$\lim _{n \rightarrow \infty}\left(\mathrm{TB} y_{n}, \mathrm{BT} y_{n}, t\right)=1$

$\Rightarrow \mathrm{M}(\mathrm{T} z, \mathrm{Bz}, t)=1 \quad$ (Since from (3))

$\Rightarrow \mathrm{T} z=\mathrm{Bz} \quad$ (since $\mathrm{M}(x, y, t)=1 \Leftrightarrow x=y)$

- To prove that $\mathrm{Sz}=z$ :

Put $x=x_{n}$ and $y=S y_{n}$ in (1), we get

$\mathrm{M}\left(\mathrm{A} x_{n}, \mathrm{BS} y_{n}, t\right) \geq \frac{1}{2}\left\{\mathrm{M}\left(\mathrm{A} x_{n}, \mathrm{TS} y_{n}, t\right)+\mathrm{M}\left(\mathrm{S} x_{n}, \mathrm{BS} y_{n}, t\right)\right\} * \max \left\{\mathrm{M}\left(\mathrm{A} x_{n}, \mathrm{~S} x_{n}, t\right), \mathrm{M}\left(\mathrm{BS} y_{n}, \mathrm{TS} y_{n}, t\right), \mathrm{M}\left(\mathrm{S} x_{n}, \mathrm{TS} y_{n}, t\right)\right\}$ 
$\mathrm{M}\left(\mathrm{A} x_{n}, \mathrm{SB} y_{n}, k t\right) \geq \frac{1}{2}\left\{\mathrm{M}\left(\mathrm{A} x_{n}, \mathrm{ST} y_{n}, t\right)+\mathrm{M}\left(\mathrm{S} x_{n}, \mathrm{SB} y_{n}, t\right)\right\} * \max \left\{\mathrm{M}\left(\mathrm{A} x_{n}, \mathrm{~S} x_{n}, t\right), \mathrm{M}\left(\mathrm{SB} y_{n}, \mathrm{ST} y_{n}, t\right), \mathrm{M}\left(\mathrm{S} x_{n}, \mathrm{ST} y_{n}, t\right)\right\}$

(Since the pairs $\{\mathrm{B}, \mathrm{S}\}$ and $\{\mathrm{S}, \mathrm{T}\}$ commute with each other).

Taking $n \rightarrow \infty$ on both sides, we have

$$
\begin{aligned}
& \mathrm{M}(u, \mathrm{~S} z, k t) \\
& \geq \frac{1}{2}\{\mathrm{M}(u, \mathrm{~S} z, t)+\mathrm{M}(u, \mathrm{~S} z, t)\} * \max \{\mathrm{M}(u, u, t), \mathrm{M}(\mathrm{S} z, \mathrm{~S} z, t), \mathrm{M}(u, \mathrm{~S} z, t)\} \quad \text { Since from (2) and (3)) } \\
& \geq \frac{1}{2}\{\mathrm{M}(u, \mathrm{~S} z, t)+\mathrm{M}(u, \mathrm{Sz}, t)\} * \max \{1,1, \mathrm{M}(u, \mathrm{~S} z, t)\} \quad(\text { Since } \mathrm{M}(x, y, t)=1 \Leftrightarrow x=y) \\
& \mathrm{M}(u, \mathrm{~S} z, k t) \geq \mathrm{M}(u, \mathrm{~S} z, t) * 1 \\
& \mathrm{M}(u, \mathrm{~S} z, k t) \geq \mathrm{M}(u, \mathrm{~S} z, t) \\
& \Rightarrow \mathrm{Sz}=u \quad(\text { from lemma (2.8)) }
\end{aligned}
$$

But from (4), we get $\mathrm{Sz}=\mathrm{z}$

- To prove $\mathrm{Bz}=z$ :

Put $x=x_{n}$ and $y=z$ in (1)

$$
\mathrm{M}\left(\mathrm{A} x_{n}, \mathrm{~B} z, k t\right) \geq \frac{1}{2}\left\{\mathrm{M}\left(\mathrm{A} x_{n}, \mathrm{~T} z, t\right)+\mathrm{M}\left(\mathrm{S} x_{n}, \mathrm{~B} z, t\right)\right\} * \max \left\{\mathrm{M}\left(\mathrm{A} x_{n}, \mathrm{~S} x_{n}, t\right), \mathrm{M}(\mathrm{B} z, \mathrm{~T} z, t), \mathrm{M}\left(\mathrm{S} x_{n}, \mathrm{~T} z, t\right)\right\}
$$

Taking limit $n \rightarrow \infty$ on both sides, we get

$\mathrm{M}(u, \mathrm{~B} z, k t) \geq \frac{1}{2}\{\mathrm{M}(u, \mathrm{~T} z, t)+\mathrm{M}(u, \mathrm{~B} z, t)\} * \max \{\mathrm{M}(u, u, t), \mathrm{M}(\mathrm{B} z, \mathrm{~T} z, t), \mathrm{M}(u, \mathrm{~T} z, t)\} \quad$ (Since from (2))

$\mathrm{M}(u, \mathrm{~B} z, k t) \geq \frac{1}{2}\{\mathrm{M}(u, \mathrm{~B} z, t)+\mathrm{M}(u, \mathrm{~B} z, t)\} * \max \{1,1, \mathrm{M}(u, \mathrm{~B} z, t)\} \quad$ (Since from (5))

$\mathrm{M}(u, \mathrm{~B} z, k t) \geq \mathrm{M}(u, \mathrm{~B} z, t) * 1$

$\mathrm{M}(u, \mathrm{Bz}, k t) \geq \mathrm{M}(u, \mathrm{Bz}, t)$

$\therefore \quad \mathrm{Bz}=u \quad$ (from lemma (2.8))

But from (4), we get $\mathrm{Bz}=\mathrm{z}$

$\therefore$ From (5), (6) and (7) we have $\mathrm{Sz}=\mathrm{Bz}=\mathrm{Tz}=\mathrm{z}$

- To prove $\mathrm{A} z=z$ :

Consider

$$
\begin{aligned}
\mathrm{M}(\mathrm{A} z, z, t) & \geq \mathrm{M}(\mathrm{A} z, \mathrm{~B} z, k t) * \mathrm{M}(\mathrm{B} z, z, t-k t) \\
& \geq \mathrm{M}(\mathrm{A} z, \mathrm{~B} z, k t) * 1 \quad(\text { from }(8) \text { and } \mathrm{M}(x, y, t)=1 \Leftrightarrow x=y) \\
& \geq \frac{1}{2}\{\mathrm{M}(\mathrm{A} z, \mathrm{~T} z, t)+\mathrm{M}(\mathrm{Sz}, \mathrm{B} z, t)\} * \max \{\mathrm{M}(\mathrm{A} z, \mathrm{~S} z, t), \mathrm{M}(\mathrm{B} z, \mathrm{~T} z, t), \mathrm{M}(\mathrm{S} z, \mathrm{~T} z, t)\}(\text { from }(1)) \\
& \left.\geq \frac{1}{2}\{\mathrm{M}(\mathrm{A} z, z, t)+\mathrm{M}(z, z, t)\} * \max \{\mathrm{M}(\mathrm{A} z, z, t), \mathrm{M}(z, z, t), \mathrm{M}(z, z, t)\} \quad \text { (Since from }(8)\right) \\
& \geq \frac{1}{2}\{M(\mathrm{Az}, z, t)+1\} * \max \{\mathrm{M}(\mathrm{Az}, z, t), 1,1\} \\
\mathrm{M}(\mathrm{A} z, z, t) & \geq \frac{1}{2}\{\mathrm{M}(\mathrm{Az}, z, t)+1\} * 1
\end{aligned}
$$




$$
\begin{aligned}
& \mathrm{M}(\mathrm{A} z, z, t) \geq \frac{1}{2}\{\mathrm{M}(\mathrm{A} z, z, t)+1\} \\
& \mathrm{M}(\mathrm{A} z, z, t) \geq \frac{1}{2} \mathrm{M}(\mathrm{A} z, z, t)+\frac{1}{2} \\
& \frac{1}{2} \mathrm{M}(\mathrm{A} z, z, t) \geq \frac{1}{2} \\
& \mathrm{M}(\mathrm{A} z, z, t) \geq 1 \\
& \therefore \mathrm{A} z=z
\end{aligned}
$$

$\Rightarrow$ from (8) and (9), we have $\mathrm{Az}=\mathrm{Sz}=\mathrm{Bz}=\mathrm{T} z=z$

$\therefore z$ is a common fixed point of $A, B, S, T$.

- To prove Uniqueness of $z$ :

Let us assume that $\mathrm{A}, \mathrm{B}, \mathrm{S}, \mathrm{T}$ have another common fixed point in $\mathrm{X}$ say $p$ where $\mathrm{Z} \neq p$

i.e., $\mathrm{A} p=\mathrm{S} p=\mathrm{B} p=\mathrm{T} p=p$

Now we prove that $z=p$.

Consider

$$
\begin{aligned}
& \mathrm{M}(z, p, k t)=\mathrm{M}(\mathrm{A} z, \mathrm{~B} p, k t) \\
& \quad \geq \frac{1}{2}\{\mathrm{M}(\mathrm{A} z, \mathrm{~T} p, t)+\mathrm{M}(\mathrm{S} z, \mathrm{~B} p, t)\} * \max \{\mathrm{M}(\mathrm{A} z, \mathrm{~S} z, t), \mathrm{M}(\mathrm{B} p, \mathrm{~T} p, t), \mathrm{M}(\mathrm{S} z, \mathrm{~T} p, t)\} \\
& \quad \geq \frac{1}{2}\{\mathrm{M}(z, p, t)+\mathrm{M}(z, p, t)\} * \max \{\mathrm{M}(z, z, t), \mathrm{M}(p, p, t), \mathrm{M}(z, p, t)\} \\
& \quad \geq \frac{1}{2}\{\mathrm{M}(z, p, t)+\mathrm{M}(z, p, t)\} * \max \{1,1, \mathrm{M}(z, p, t)\} \\
& \geq \mathrm{M}(z, p, t) * 1 \\
& \quad \geq \mathrm{M}(z, p, t) \\
& \mathrm{M}(z, p, k t) \geq \mathrm{M}(z, p, t)
\end{aligned}
$$

$\therefore z=p \quad$ (using lemma (2.8))

$\therefore$ There exists a unique common fixed point of $\mathrm{A}, \mathrm{B}, \mathrm{S}, \mathrm{T}$ in $\mathrm{X}$.

Example 3.2: Let $\mathrm{X}=[0,2], \mathrm{M}(x, y, t)=\mathrm{e}^{\frac{-d(x, y)}{t}}(0<t<1)$ where $d(x, y)=|x-y|$ and $*$ be the continuous t-norm given by $a * b=\min \{a, b\}$.

Clearly, $(\mathrm{X}, \mathrm{M}, *)$ is a Complete fuzzy metric space.

Let $\mathrm{A}=1, \mathrm{~B}=\frac{2 x+5}{7}, \mathrm{~S}=x, \mathrm{~T}=\frac{3 x+1}{4}$ be self maps on $\mathrm{X}$.

Let $\left\{x_{n}\right\}=\left\{1-\frac{1}{n}\right\}_{n \in \mathrm{N}} \quad$ be a sequence in $\mathrm{X}$.

$\lim _{n \rightarrow \infty} \mathrm{SA} x_{n}=\mathrm{S}(1)$ and $\lim _{n \rightarrow \infty} \mathrm{A} x_{n}=\mathrm{A}(1)$

$\lim _{n \rightarrow \infty} \mathrm{A} x_{n}=1$ and $\lim _{n \rightarrow \infty} \mathrm{S} x_{n}=1$ where $1 \in \mathrm{X}$.

$\Rightarrow \mathrm{A}$ and $\mathrm{S}$ are reciprocally continuous.

Let $\left\{y_{n}\right\}=\left\{1+\frac{1}{n}\right\}_{n \in \mathrm{N}} \quad$ be a sequence in $\mathrm{X}$.

$\lim _{n \rightarrow \infty}\left(\mathrm{TB} y_{n}, \mathrm{BT} y_{n}, t\right)=1$ and $\lim _{n \rightarrow \infty} \mathrm{B} y_{n}=\lim _{n \rightarrow \infty} \mathrm{T} y_{n}=1$ where $1 \in \mathrm{X}$.

$\Rightarrow \mathrm{B}$ and $\mathrm{T}$ are $\mathrm{z}$-asymptotically commuting where $\mathrm{z}=1$.

Also, the four maps satisfies (iii) and (iv) of theorem 3.1.

$\Rightarrow \mathrm{A}, \mathrm{B}, \mathrm{S}, \mathrm{T}$ have a Unique common fixed point in $\mathrm{X}$ i.e., at $x=1$. 


\section{References}

[1] Zadeh, L.A. (1965) Fuzzy Sets. Information and Control, 8, 338-353. http://dx.doi.org/10.1016/S0019-9958(65)90241-X

[2] Kramosil, L. and Michalek, J. (1975) Fuzzy Metric and Statistical Metric Spaces. Kybernetica, 15, 326-334.

[3] George, A. and Veeramani, P. (1994) On Some Results in Fuzzy Metric Spaces. Fuzzy Sets and Systems, 64, 395-399. http://dx.doi.org/10.1016/0165-0114(94)90162-7

[4] George, A. and Veeramani, P. (1997) On Some Results of Analysis for Fuzzy Metric Spaces. Fuzzy Sets and Systems, 90, 365-368. http://dx.doi.org/10.1016/S0165-0114(96)00207-2

[5] Mihet, D. (2004) A Banach Contraction Theorem in Fuzzy Metric Spaces. Fuzzy Sets and Systems, 144, 431-439. http://dx.doi.org/10.1016/S0165-0114(03)00305-1

[6] Aage, C.T. and Salunke, J.N. (2010) On Fixed Point Theorems in Fuzzy Metric Spaces. International Journal of Open Problems in Computer Science and Mathematics, 3, 123-131.

[7] Kailash, N., Rajesh, S., Manoj, S. and Manoj, S. (2013) Common Fixed Point Theorem for Four Mappings in Fuzzy Metric Space. Research Journal of Mathematical and Statistical Sciences, 1, 11-14.

[8] Manandhar, K.B., Jha, K. and Pathak, H.K. (2014) A Common Fixed Point Theorem for Compatible Mappings of Type(E) in Fuzzy Metric Space. Applied Mathematical Sciences, 8, 2007-2014.

[9] Badshah, V.H., Rathore, G.P.S. and Katlana, P. (2014) Common Fixed Point Theorems in Fuzzy Metric Space Using Occasionally Weakly Compatible Mappings. International Journal of Theoretical and Applied Sciences, 6, 62-64.

[10] Balasubramaniam, P., Muralisankar, S. and Pant, R.P. (2002) Common Fixed Points of Four Mappings in a Fuzzy Metric Space. Journal of Fuzzy Mathematics, 10, 379-384.

[11] Mishra, S.N., Sharma, N. and Singh, S.L. (1994) Common Fixed Points of Maps on Fuzzy Metric Spaces. International Journal of Mathematics and Mathematical Sciences, 17, 253-258. http://dx.doi.org/10.1155/S0161171294000372

[12] Tivari, B.M.L. and Singh, S.L. (1986) A Note on Recent Generalizations of Jungck Contraction Principle. Journal. Uttar Pradesh Government Colleges Academic Society, 3, 13-18.

[13] Schweizer, B. and Sklar, A. (1960) Statistical Metric Spaces. Pacific Journal of Math., 10, 313-334. http://dx.doi.org/10.2140/pjm.1960.10.313

[14] Grabiec, M. (1988) Fixed Points in Fuzzy Metric Spaces. Fuzzy Sets and Systems, 27, 385-389. http://dx.doi.org/10.1016/0165-0114(88)90064-4 